\section{Absorption of Hydrogen Lyman Radiation by Atmospheric Gases}

THE absorption of the hydrogen resonance line at $1215 \cdot 7 \mathrm{~A}$. has been investigated to obtain more exact information bearing on the theory of the production of short-wave radio fadeouts by solar eruptions ${ }^{1}$. The following results have been obtained :

(1) Nitrogen is transparent in layers up to $3 \cdot 2 \mathrm{~mm}$. thick (N.T.P.), in agreement with the early observations of Hopfield ${ }^{2}$ and more recent measurements by Preston $^{3}$.

(2) Exact measurements on water vapour were difficult, but more than half the incident radiation is absorbed in a layer about $0.05 \mathrm{~mm}$. thick. This agrees with Preston's observations.

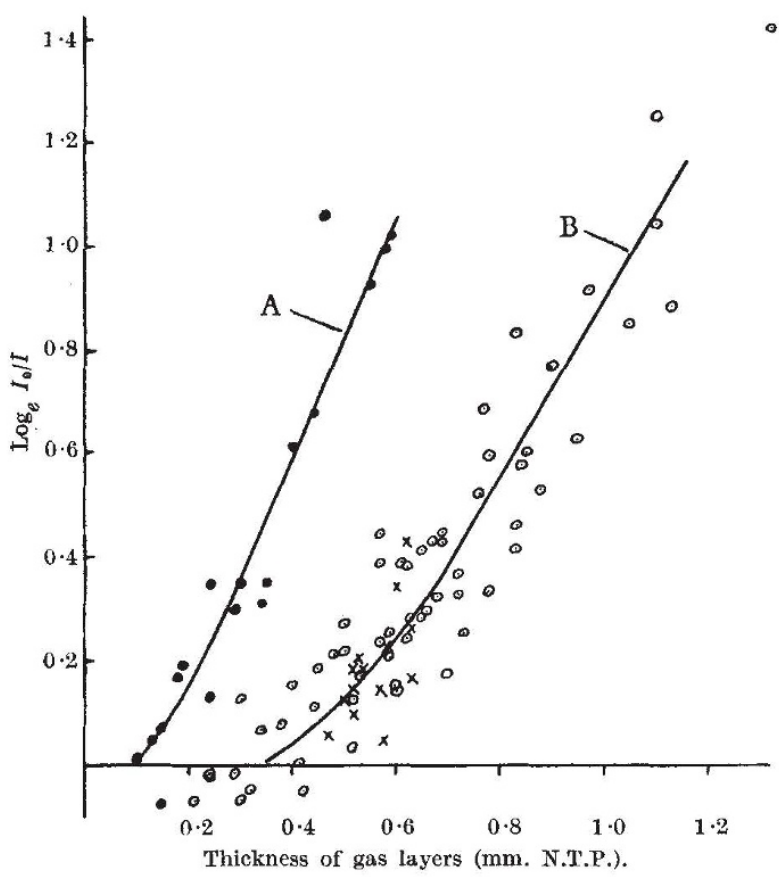

- oxygen; $\odot$, air (energy in disruptive charge, 6 watts) $x$, air (energy in disruptive charge, 3 watts).

(3) The absorption in oxygen and air was found to be much heavier than was expected from previous work. In the region where absorption commences, the densities of the parts of the hydrogen line in the absorption spectrum and the check spectrum without absorber, which was put on the same film, were compared directly as an approximate measure of the absorption. Lack of the characteristic curves for the emulsion should not cause serious errors in the comparison of nearly equal densities.

In the accompanying figure, Curve $A$ shows the absorption of molecular oxygen in layers up to $0.6 \mathrm{~mm}$. thick (N.T.P.). The ordinates are $\log _{e} I_{0} / I$ ( $I_{0}$ being the unabsorbed and $I$ the absorbed intensity); in the usual formula for mass absorption. $\log _{e} I_{0} / I=\mu x+c$. Since absorption appears to start only when there is a minimum gas layer amounting to about $0.10 \mathrm{~mm}$., then $c$ is not, in this case, zero In addition, the absorption coefficient, $\mu$, mav not be constant for small values of the absorption.
Curve $B$ shows the absorption in air. Several points falling below the axis of zero absorption indicate the possible magnitude of individual errors, and the general scatter of observations makes it difficult to define the absorption curve accurately. Its slope, however, is not constant, and again, there is no absorption until a minimum layer of gas is present. This minimum appears to be between $0 \cdot 30$ and $0.40 \mathrm{~mm}$. (N.T.P.) and is not exactly five times the minimum in oxygen. For the strong bands at 1140,1245 and $1450 \mathrm{~A}$. absorption is clearly apparent in the least amounts of gas used for observations on $1215 \cdot 7 \mathrm{~A}$.

The absorption coefficients given for oxygen and air by Preston are about fifty times less. In the present case the absorbing gases were pure to within three parts in a thousand, and, in addition, water vapour, the most powerful absorber, was ruled out as an unobserved impurity by check experiments with saturated nitrogen. It is possible that Preston's observations were in error owing to serious dissociation of the oxygen in his apparatus (cf. the difficulties experienced by Ladenburg and van Voorhis ${ }^{4}$ ). In the present case reduction to half the energy dissipated in the discharge caused no observable difference in the absorption measurements, showing that dissociation of the oxygen was negligible.

With a fixed absorption path the pressure is varied in order to change the thickness of the absorbing layer, and it would appear from the two curves, therefore, that the partial pressure of the oxygen in the spectrograph is the factor determining the onset of absorption. This minimum pressure is about $0.04 \mathrm{~mm}$. If absorption of $1215.7 \mathrm{~A}$. is only possible when an oxygen molecule is in a slightly perturbed state owing to its collision with another oxygen molecule, the absorption coefficient should vary as the square of the pressure until the population density of absorbing molecules is constant. In oxygen at very high pressures, Shalow and Steiner ${ }^{5}$ have found absorption bands which behave in this way, and attributed by them to the temporary formation of $\mathrm{O}_{2}-\mathrm{O}_{2}$ molecules.

If $\lambda 1215 \cdot 7$ is emitted from the sun, no absorption will take place until it reaches levels in the upper atmosphere where the partial pressure of molecular oxygen is about $0.04 \mathrm{~mm}$. (about $80 \mathrm{~km}$.), except for attenuation due to the small amount of water-vapour above this height. According to the shape of the 'tail' of the absorption curve, the radiation would be absorbed more or less rapidly in dissociating oxygen molecules and producing ionization according to the process previously suggested $^{1}$. The resultant ionized layer would perhaps correspond to that found by Budden, Ratcliffe and Wilkes 6 .

This work has been made possible through a grant for research made by the Commonwealth Government.

\section{S. E. Wilutams.}

School of Physics,

University of Sydney, N.S.W. Nov. 30

${ }^{1}$ Martyn, Munro, Higgs and Williams, Nature, 140, 603 (1937).

'Hopfleld, Phys. Rev., 20, 573 (1922).

a Preston, Phys. Rev., 55, 1125, 1939 (abstract only). An attempt to communicate with $\mathrm{Mr}$. Preston privately regarding the discrepancy was unsuccessful.

1 Ladenburg and van Voorhis, Phys. Rev., 43, 315 (1933).

'Shalow and Steiner, Z. Phys., 99, 137 (1936).

'Budden, Ratcliffe and Wilkes, Proc. Roy. Soc., A, 171, 188 (1939). 